\title{
Iterative optimierungsbasierte Simulation
}

\author{
Entscheidungsunterstützung in der hierarchischen Planung \\ komplexer Produktions- und Logistiksysteme
}

\author{
Patrick Oetjegerdes, Christian Weckenborg und Thomas S. Spengler, Technische Universität Braunschweig
}

\begin{abstract}
Werden Anpassungen von Produktionssystemen geplant, beispielsweise der Bau einer neuen Anlage, werden häufig Simulationen für die Prognose der damit verbundenen Auswirkungen genutzt. So wird die Basis für eine betriebswirtschaftliche Bewertung geschaffen. Für die Erstellung der Modelle werden Entscheidungsprozesse der Produktionsplanung und -steuerung in der Simulation dargestellt, beispielsweise die Reihenfolgenplanung einer Anlage. Diese Übertragung der Entscheidungsprozesse in die Simulation führt zu einem hohen Aufwand für die Erstellung von Simulationsmodellen. Dieser Aufwand kann durch die Nutzung iterativer optimierungsbasierter Simulationen verringert werden, da hier auf bereits existierende Prozesse zurückgegriffen wird. Statt Entscheidungsprozesse in der Simulation neu zu modellieren und lösen, wird die Simulation mit Optimierungsverfahren gekoppelt, die auch im realen System zur Entscheidungsfindung genutzt werden. Dieses Konzept wird bei einem Stahlhersteller angewendet und es wird gezeigt, dass so verschiedene operative bis taktische Anpassungen bewertet werden können.
\end{abstract}

Verschiedene interne und externe Faktoren führen dazu, dass Unternehmen ihre strategische Planung anpassen. Beispiele sind die Verfügbarkeit neuer Technologien oder Änderungen des Marktumfelds. Diese Anpassung der strategischen Unternehmensplanung bedingt häufig Anpassungen der Produktionssysteme und damit der Produktionsplanung.

Auf der strategischen Ebene wird häufig über das grundlegende Portfolio angebotener Produkte oder Dienstleistungen und ihre Zuordnung zu Produktionsstandorten entschieden. Die Erbringung dieser Leistungen muss gemäß dem Prinzip der hierarchischen Planung auf taktischer und operativer Ebene ermöglicht werden.

\section{Herausforderungen strategischer Produktionsplanung}

Folglich sind im Rahmen der Produktionsplanung verschiedene Fragestellungen zu adressieren:

- Wo entsteht der Engpass im System?

- Ist der Engpass weit genug, um die strategische Zielsetzung zu erreichen?

- Sind weitere operative (z. B. eine geänderte Reihenfolgenplanung) und taktische Anpas- sungen (z. B. Investitionen in weitere, kleinere Anlagen) notwendig, um die strategische Zielsetzung zu erreichen?

- Welche Anpassungsbündel zur Erfüllung der strategischen Zielsetzung sind betriebswirtschaftlich optimal?

Um diese Fragen beantworten zu können, ist es zunächst notwendig, die Auswirkungen der Anpassung der strategischen Unternehmensplanung auf das Produktionssystem zu prognostizieren. Da es in komplexen Produktionssystemen nicht möglich ist, diese Auswirkungen analytisch zu bestimmen, wird hierzu häufig die ereignisdiskrete Simulation (fortan als Simulation bezeichnet) genutzt. [1] Die Erstellung eines geeigneten Simulationsmodells ist jedoch komplex.

Es ist notwendig, dass die operative und taktische Planung nicht vernachlässigt werden. Gemäß dem Prinzip der hierarchischen Planung wird die grobe Planung der strategischen Ebene sukzessiv auf ihre Durchführbarkeit auf den detaillierteren taktischen und operativen Ebenen überprüft. [2] Folglich ist es notwendig, diese Überprüfung in der Simulation nachzubilden und die taktische und operative Planung in der Simulation darzustellen.
Iterative Optimization-based Simulation Decision Support for Adjustments in ComSimulation is frequently used for prediction of the simulation. To achieve this, complex real processes have to be transferred into creation of simulation models. This is rosolved concept of iterative optimization-based parameters are exported and existing decision eywords: planning, discrete-event simulation, producon facility planning

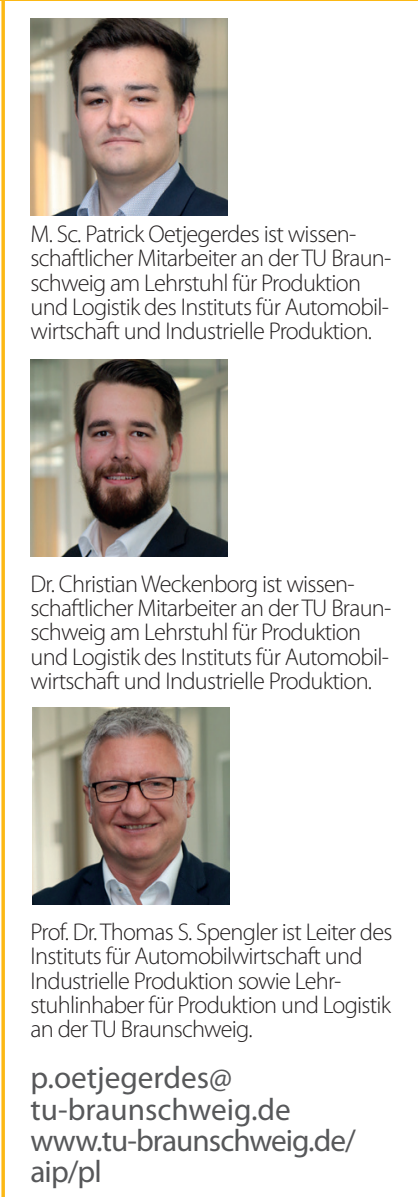


Die Herausforderung besteht also darin, eine Simulation eines Produktionssystems zu erstellen, die in der Lage ist strategische Entscheidungen ebenso wie die Auswirkungen taktischer und operativer Maßnahmen abzubilden.

Iterative optimierungsbasierte Simulation in der Theorie

Bei der Unterstützung strategischer Entscheidungen durch die Simulation eines Produktionssystems ergeben sich einige Herausforderungen. Während der Laufzeit der Simulation, welche die Reichweite der strategischen Entscheidungen abbildet, müssen eine Reihe taktischer und operativer Entscheidungen getroffen werden. Beispiele hierfür sind Kapazitätszuweisungen von Anlagen oder Verladeprobleme auf Transportfahrzeuge. Diese Planungsprobleme treten auch in der Simulation auf. Typischerweise werden diese in der Simulation durch einfache Heuristiken gelöst, beispielsweise Prioritätsregeln, während in der Realität komplexe Optimierungsverfahren genutzt werden, welche in Produkten des Enterprise Resource Planning und der Manufacturing Execution Systems vorliegen. Somit liegt eine Diskrepanz zwischen der realen Planung und ihrer simulationsbasierten Abbildung vor. Um eine hohe Realitätsnähe der Simulation zu ermöglichen, können die genutzten Optimierungsverfahren in dedizierter Software abgebildet werden. Idealerweise wird die selbe Software genutzt, wie in der Realität, sodass vorhandene Schnittstellen genutzt werden können. Somit ergibt sich eine Koppelung von Simulation und Optimierung.

Es werden grundsätzlich zwei Varianten unterschieden: die sequentielle und die hierarchische Kopplung. Bei einer sequentiellen Koppelung läuft erst die Simulation komplett ab und die Optimierung folgt (oder vice versa). Bei der hierarchischen Koppelung kann entweder die Optimierung oder die Simulation die Leitkomponente darstellen, welche die jeweils andere während ihrer Laufzeit mehrfach aufruft. [3]

Bei der simulationsbasierten Optimierung gibt die optimierende Leitkomponente Werte der Entscheidungsvariablen an die Simulation, die hieraus die Qualität der Lösung bestimmt. Diese wird von der optimierenden Komponente erneut aufgegriffen. In der optimierungsbasierten Simulation ist die Simulation die Leitkomponente und während der Laufzeit der Simulation werden Teilplanungsprobleme an eine optimierende Komponente übergeben, von dieser gelöst und die Ergebnisse von der Simulation in der weiteren Durchführung des
Simulationslaufs genutzt. [3] Verschiedene praxisnahe Anwendungsbeispiele der hierarchischen Kopplung von Simulation und Optimierung finden sich in [4].

Zusätzlich zu der Art der Kopplung, können Simulationen nach ihrem Zweck unterschieden werden. Die Simulation kann entweder dazu genutzt werden einen Zielfunktionswert zu bestimmen, der die Optimierung steuert (Evaluierungsfunktion) oder als Modell genutzt werden, in dem die Optimierung eine Variablenausprägung bestimmt, welche anschließend auf die Realität übertragen werden kann (Ersatzmodellfunktion). Auch ist es möglich die Simulation zu nutzen, um die Optimierung zu erweitern und zu verbessern, indem z.B. stochastische Elemente durch die Simulation eingebracht werden (Modellerweiterungsfunktion). Auch ist es möglich, dass die Simulation verschiedene Parameter berechnet, die zur Entscheidungsunterstützung benötigt werden, für deren Bestimmung aber auf optimierende Verfahren zurückgegriffen werden muss (Lösungsgenerierungsfunktion) [5].

Im vorliegenden Anwendungsfall der strategischen Produktionsplanung wird die Simulation genutzt, um zu überprüfen, ob die strategische Planung umsetzbar ist. Es ist also notwendig verschiedene Kennzahlen zu bestimmen, die abbilden, ob das Produktionssystem überlastet ist. Die Simulation erfüllt damit die Lösungsgenerierungsfunktion. Die Koppelung von Simulation und Optimierung erfolgt in dem Anwendungsfall hierarchisch mit der Simulation als Leitkomponente. Während der Laufzeit ist es notwendig operative und taktische Entscheidungen zu treffen, die mithilfe eines optimierenden Verfahrens bestimmt werden. Es liegt somit eine optimierungsbasierte Simulation vor. Der Zweck der Simulation ist die Lösungsgenerierung. Die resultierende Klasse simulationsbasierter Methoden wird als iterative optimierungsbasierte Simulation bezeichnet. [5] Bisherige Arbeiten beschränken sich hierbei auf die Übertragung einzelner Entscheidungen auf optimierende Verfahren und auf operative Entscheidungen. Um dieses Prinzip jedoch auf die hierarchische Planung zu übertragen, muss der Einbezug mehrerer, operativer und taktischer, Entscheidungen ermöglicht werden.

Iterative optimierungsbasierte Simulation in der hierarchischen Produktionsplanung

In der hierarchischen Planung wird zunächst das Teilplanungsproblem mit dem längsten Zeithorizont mit starken Vereinfachungen ge- 

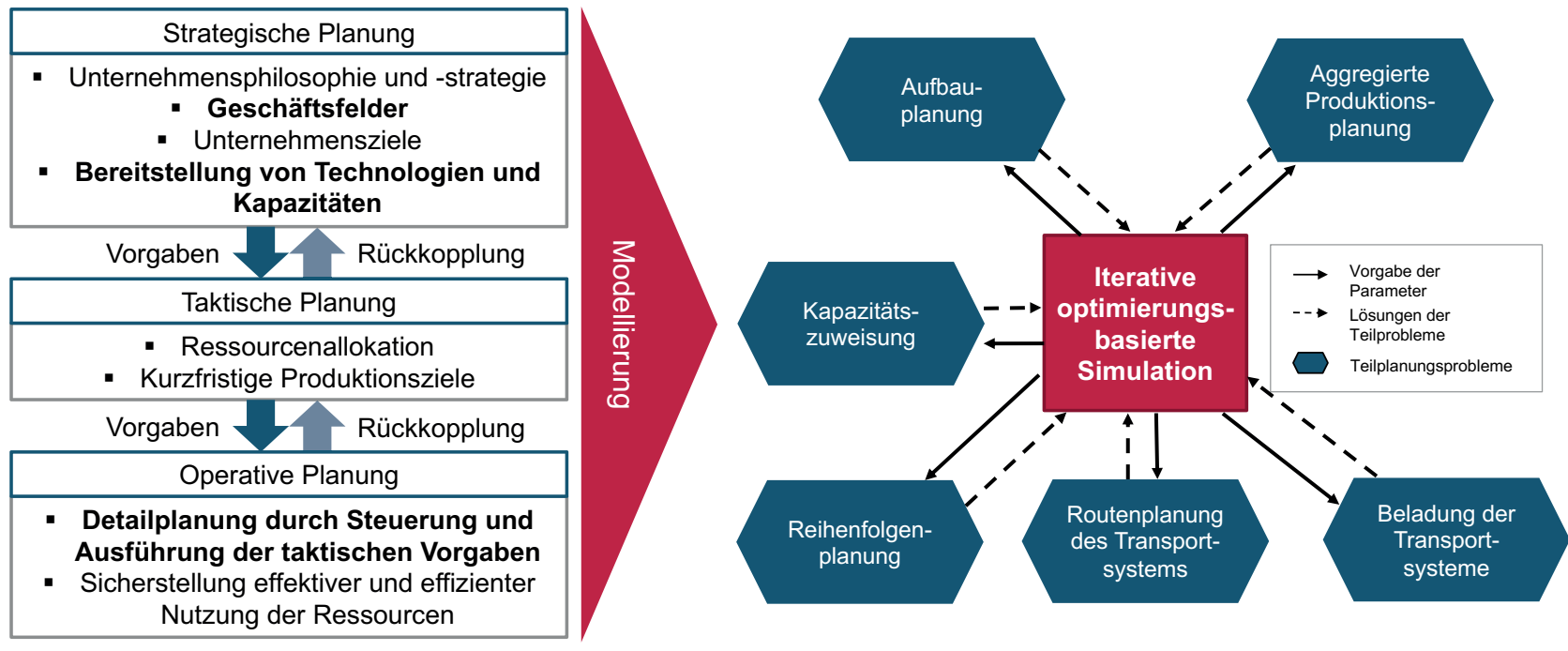

Iöst. Die resultierenden Parameter stellen dann eine Zielvorgabe für das Teilplanungsproblem auf der nächstdetaillierteren Stufe mit einem kürzeren Zeithorizont dar. Ist diese Vorgabe nicht umsetzbar, werden die durch das übergeordnete Teilplanungsproblem aufgeprägten Restriktionen gelockert. Ist das untergeordnete Teilplanungsproblem nun lösbar, werden die resultierenden Parameter an das nächstdetailliertere Teilplanungsproblem mit erneut kürzerem Zeithorizont weitergegeben. Dieses Vorgehen wird so lange weitergeführt, bis auch alle operativen Teilplanungsprobleme auf Basis der strategischen Zielsetzung lösbar sind und die strategische Planung somit umsetzbar ist. [2]

Dieses Prinzip wird nun aufgegriffen. In der Simulation werden zu verschiedenen Zeitpunkten Parameter bestimmt und exportiert, beispielsweise Produktmengen, Auftragsdaten oder die Verfügbarkeit von Betriebsmitteln, die für die Optimierung notwendig sind. Anschließend werden diese Parameter als Vorgabe von den Teilplanungsproblemen genutzt, um ihre Lösung mithilfe der Optimierung zu bestimmen. So wird beispielsweise wöchentlich die Kapazitätszuweisung vorgenommen, einmal pro Schicht die Reihenfolgenplanung und jedes Mal bei Eintritt eines Transporters die optimale Beladung. Die Lösungen der Teilplanungsprobleme werden anschließend an die Simulation zurückgegeben und im weiteren Verlauf genutzt. Das Prinzip der hierarchischen Planung sowie die Übertragung auf die iterative optimierungsbasierte Simulation werden in Bild 1 dargestellt.

Das eingeführte Vorgehen bietet mehrere Vorteile. Zunächst einmal wird ein realitätsnahes Simulationsmodell erzeugt, indem die in der Realität vorliegenden Verfahren zur Entscheidungsfindung integriert werden. Des Weiteren wird der Aufwand der Modellierung verringert, da die Konzeption, Entwicklung und Implementierung von Heuristiken zur Abbildung des Entscheidungsfindungsprozesses entfällt. Weiterhin vereinfacht die Verwendung der existierenden Prozesse die Validierung, sofern die Implementierung verifiziert wurde. Die Verifizierung stellt sicher, dass die eingesetzten Optimierungsverfahren korrekt verknüpft wurden. Die Validierung komplexer Modelle ist in der Regel aufwendig, da sichergestellt werden muss, dass das Verhalten des verifizierten Modells der Realität entspricht. Durch die Verwendung der realen Optimierungsverfahren, kann davon ausgegangen werden, dass dies der Fall ist. Im Folgenden wird die vorgestellte Methodik auf einen Anwendungsfall in der Stahlindustrie übertragen.

\section{Iterative optimierungsbasierte Simu- lation in der Praxis}

In diesem Praxisbeispiel wird im Rahmen der strategischen Planung entschieden, eine neue Anlage zur Verzinkung von Flachstahl in ein existierendes Produktionssystem einzufügen. Die Anlage kann jedoch nicht isoliert betrachtet werden, da nachfolgende und vorangehende Anlagen ebenfalls stärker belastet werden. Dies resultiert in einem angepassten Produktionsprogramm. Ziel ist es mit dieser neuen Anlage den Output des Produktionssystems um 20 \% zu erhöhen. Zu prüfen ist, ob das Produktionsprogramm produziert werden kann.

Hierfür wurde eine rund $125.000 \mathrm{~m}^{2}$ große Produktionshalle modelliert, in der zu jedem Zeitpunkt rund 8.000 kundenindividuelle Stahlcoils in verschiedenen Produktionsstufen lagern. Diese werden in unterschiedlicher Reihenfolge von mehreren Anlagen bearbeitet, anschließend verpackt und per Zug, Coiltransporter
Bild 1: Übertragung des Prinzips der hierarchischen Planung auf die iterative optimierungsbasierte Simulation. 


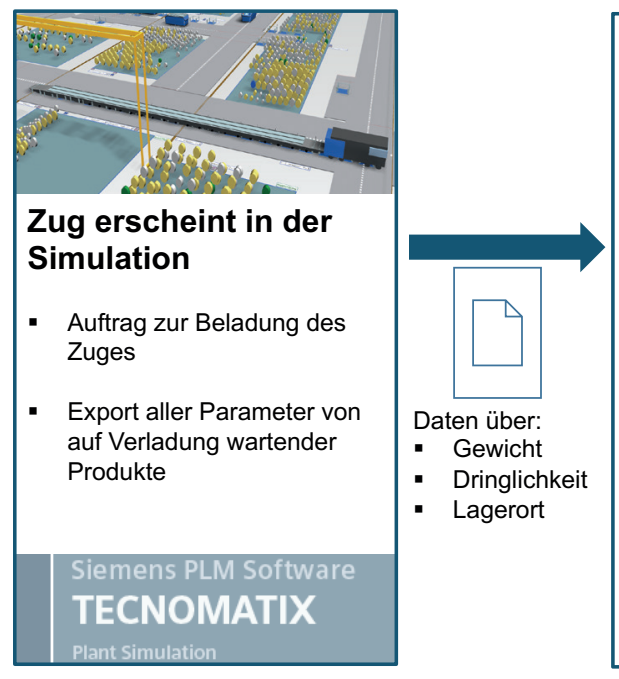

TECNOMATIX

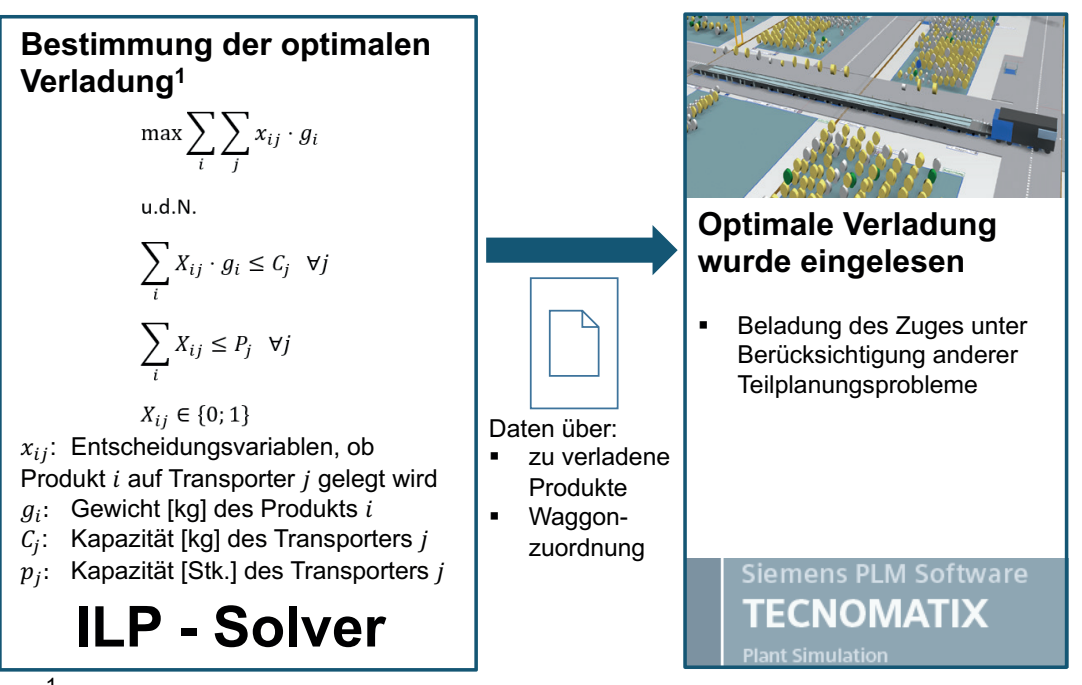

${ }^{1}$ Vereinfachtes Modell zur Veranschaulichung
Bild 2: Ablauf der Lösung eines Teilplanungsproblems während des Simulationslaufs.

\section{Literatur}

[1] Law, A.: Simulation modeling and analysis, 5. Auflage. New York 2015

[2] Steven, M.: Hierarchische Produktionsplanung, 2. Auflage Heidelberg 1994.

[3] Verein Deutscher Ingenieure: VDI-Richtlinie 3633 Blatt 12: Simulation von Logistik-, Materialfluss- und Produktionssystemen. Berlin 2020.

[4] März, L; Krug, W; Rose, O.; Weigert, G. (Hrsg): Simulation und Optimierung in Produktion und Logistik, 1. Auflage. Berlin Heidelberg 2011

[5] Figueira, G.; Almada-Lobo, B. Hybdrid simulation-optimization methods: A taxonomy and discussion. In: Simulation Modelling Practice and Theory 46 (2014), S. 118-134. oder LKW abtransportiert. Innerhalb der Produktionshalle werden sie durch Portalkräne, fahrerlose Transportsysteme, Quertransporter oder Coiltransporter transportiert. Der Betrachtungszeitraum entspricht dem Planungshorizont für das Produktionsprogramm, der sechs Wochen lang ist. Die Laufzeit für die Simulation dieses Zeitraums beträgt ungefähr eine Stunde pro Simulationslauf und ist damit für die Entscheidungsunterstützung der strategischen Planung angemessen.

Ausgangspunkt der Simulation ist die reale Lagerbelegung auf Basis des Materialverfolgungssystems. Das Materialverfolgungssystem erfasst in Echtzeit die exakte Position jedes einzelnen Produkts in der Halle, inklusive des Bearbeitungsstands. Zusätzlich werden Daten über physikalische Größen, wie Gewicht und Geometrie erfasst, sowie Auftragsdaten. Diese Daten können innerhalb weniger Minuten in die Simulation importiert werden. Während des Simulationslaufs werden unterschiedliche Teilplanungsprobleme auf Basis dieser Daten gelöst. So wird die Reihenfolge der Produktion in jeder Anlage am Anfang einer Schicht geplant. Die Routen der fahrerlosen Transportfahrzeuge werden stündlich geplant. Jedes Mal, wenn ein Stahlcoil transportiert werden soll, wird mit einer Einlagerungsheuristik der beste Lagerplatz für dieses Stahlcoil auf Basis der aktuellen Lagerbelegung und den Eigenschaften des Stahlcoils bestimmt. Durch den Einbezug realer Entscheidungsprozesse wird die Ableitung realitätsnaher Ergebnisse ermöglicht.

Der letzte Produktionsschritt einiger Coils in der Halle stellt die Verladung der gefertigten Coils zum Transport auf Züge dar. Anhand dieses Ablaufs wird die Kopplung der hierarchischen Simulation an ein optimierendes Verfahren zur Abbildung der Entscheidungsprozesse erläutert. Züge bestehen aus bis zu 10 Waggons, die jeweils eine stückbasierte und gewichtsbasierte Kapazitätsbegrenzung besitzen. Ein Großteil der Produkte muss mithilfe der Züge in eine weiter entfernte Halle transportiert werden. Ziel ist die optimale Auslastung der Züge hinsichtlich der transportierten Masse. Um dies zu erreichen wird jedes Mal, wenn ein Zug zur Beladung bereitsteht, eine Liste aller auf die Verladung wartenden Coils exportiert. Die optimale Zuordnung der Produkte auf die einzelnen Waggons wird mit einer Software für die Lösung mathematischer Optimierungsprobleme berechnet. Diese Zuweisung wird anschließend an die Simulation zurückgegeben. Nun wird auf Basis der aktuellen Lagerbelegung eine Reihenfolge zur Auslagerung mithilfe der Portalkräne bestimmt. Dieser Prozess ist in Bild 2 veranschaulicht.

\section{Fazit und Ausblick}

Die Einschätzung über die Vorteilhaftigkeit des Vorgehens basiert auf der Anwendung in einem Unternehmen mit einer umfangreichen Verfügbarkeit von Daten, welche für die Umsetzung der iterativen optimierungsbasierten Simulation für notwendig erachtet wird. Mithilfe dieses Vorgehens ist es möglich die Auswirkungen strategischer, taktischer und operativer Entscheidungen darzustellen. So wird eine Entscheidungsunterstützung für die Bewertung von Anpassungsmaßnahmen im Zusammenspiel von Teilplanungsproblemen verschiedener Fristigkeit ermöglicht.

\section{Schlüsselwörter:}

Optimierungsbasierte Simulation, Produktionsplanung, Ereignisdiskrete Simulation, Anlagenplanung 